\title{
Circular Dichroism of DNA G-Quadruplexes: Combining Modeling and Spectroscopy to Unravel Complex Structures.
}

Hugo Gattuso a,b, Angelo Spinello ${ }^{\mathrm{c}}$, Alessio Terenzi ${ }^{\mathrm{c}, \mathrm{d}}$, Xavier Assfeld ${ }^{\mathrm{a}, \mathrm{b}}$, Giampaolo Barone ${ }^{\mathrm{c}}$, and Antonio Monari ${ }^{\mathrm{a}, \mathrm{b}}$

${ }^{a}$ Universite de Lorraine Nancy, Theory-Modeling-Simulation, SRSMC, Boulevard des Aiguillettes 54506, Vandoeuvre-les-Nancy, France

${ }^{\mathrm{b}}$ CNRS, Theory-Modeling-Simulation, SRSMC, Boulevard des Aiguillettes 54506, Vandoeuvre-les-Nancy, France

${ }^{c}$ Universita di Palermo, Dipartimento di Scienze Biologiche, Chimiche e Farmaceutiche, Viale delle Scienze, Palermo Italy

${ }^{\mathrm{d}}$ Institute of Inorganic Chemistry University of Vienna Wahringerstrasse 42 Vienna, Austria

I) Circular dichroism with different QM partitions.

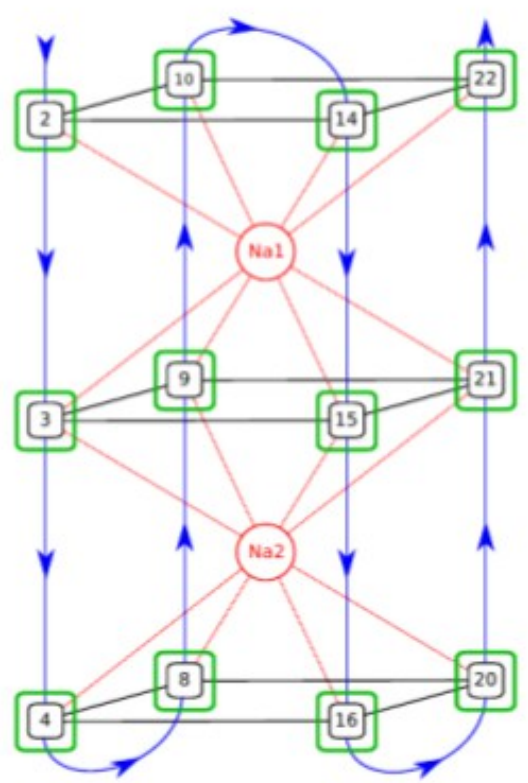

A) Bases

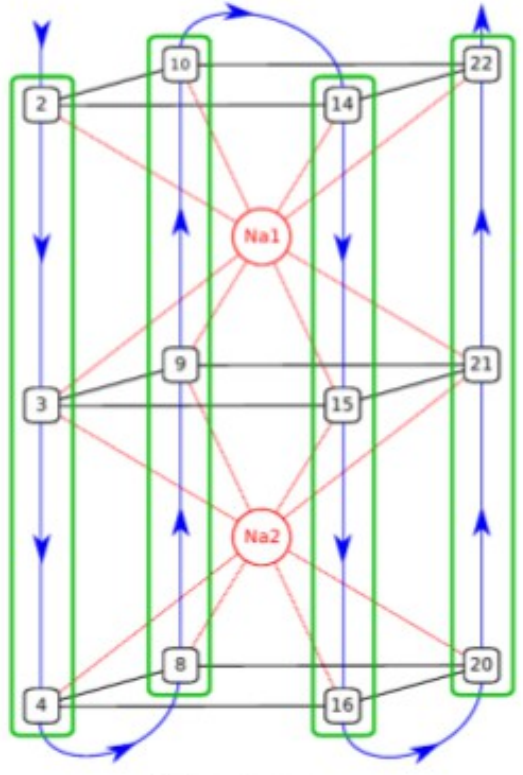

B) Rows

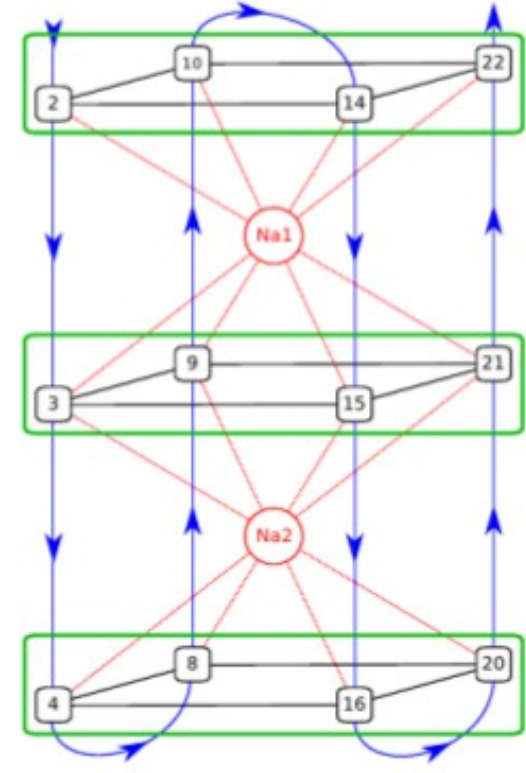

C) Planes

Figure S1: Schematic representation of the 3 QM units schemes tested in this study. Each green box represents one of the monomeric QM units used to build Frenkel hamiltonian. 

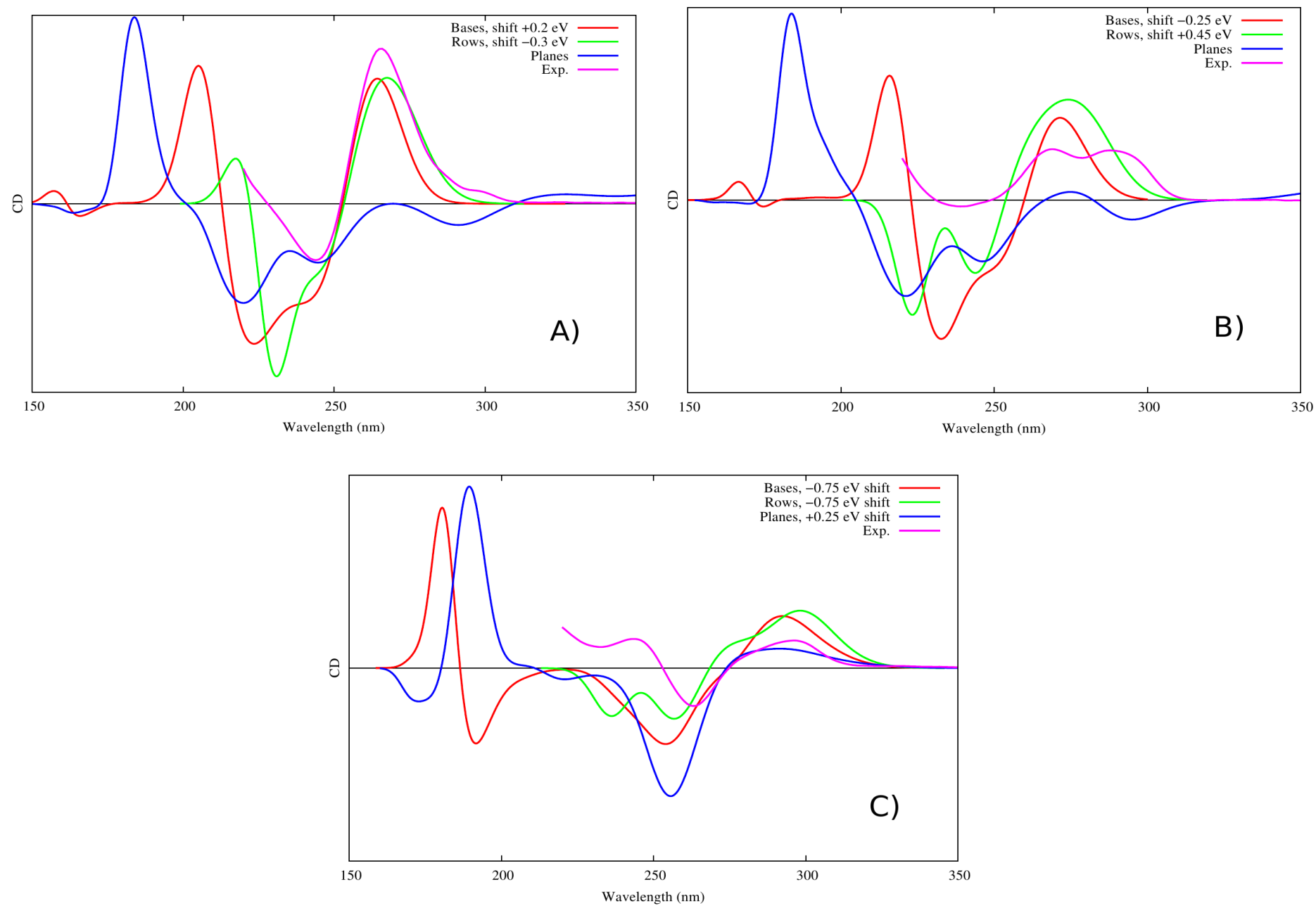

Figure S2: Computed and experimental circular dichroism spectra for each QM partition scheme as presented in Figure S1. A) Parallel, B) Hybrid and C) Antiparallel. Bases alone (S1 A, red), rows of 3 bases (S1 B, green), planes of 4 bases (S1 C, blue) and experimental (purple). Each computed spectra has been shifted by a value indicated on the figure to fit with the experimental spectra. The best agreement between computed and experimental spectra for the 3 G4 types is found for the rows QM units. This can be explained by the fact that some excited states are delocalized through the stacked nucleobases. ${ }^{1}$ 
II) Full circular dichroism spectrum
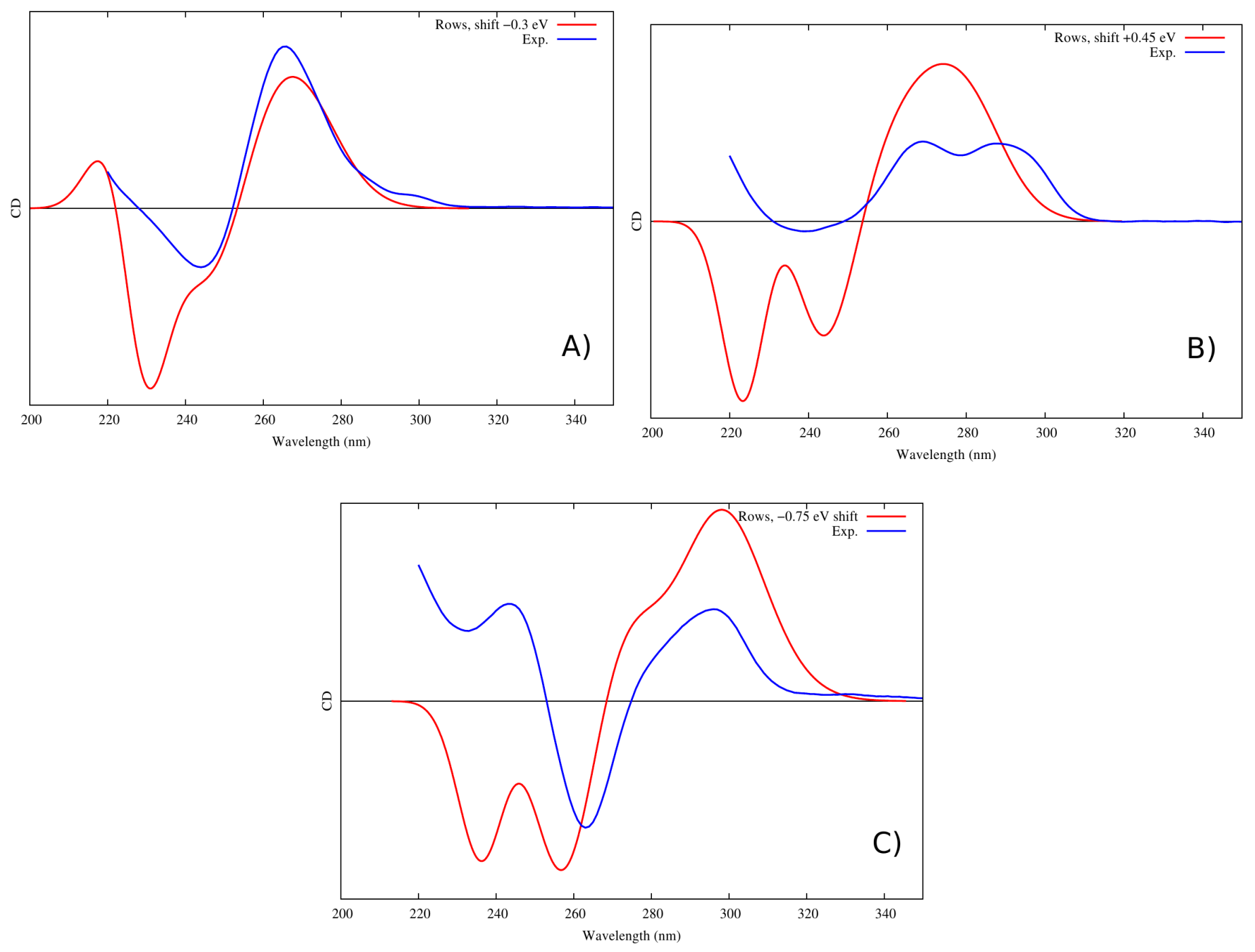

Figure 3: Computed (red) and experimental (blue) spectra of A) Parallel, B) Hybrid and C) Antiparallel including higher energy transitions 
III) CD spectra of the hybrid G4 for different width of the convolution gaussians.

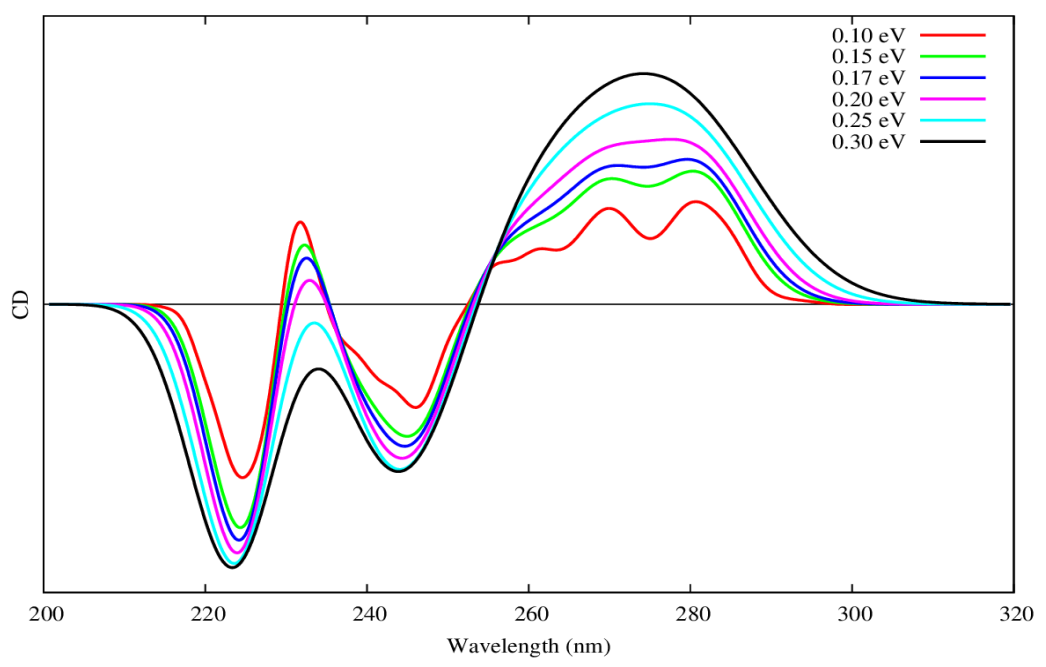

Figure 4: Circular dichroism spectra of hybrid G4 with different width of the convolution gaussians. It is noticeable that the two characteristic positive bands of the hybrid type appear when the peak width decreases. 
IV)Effect of the environment on the CD spectra.
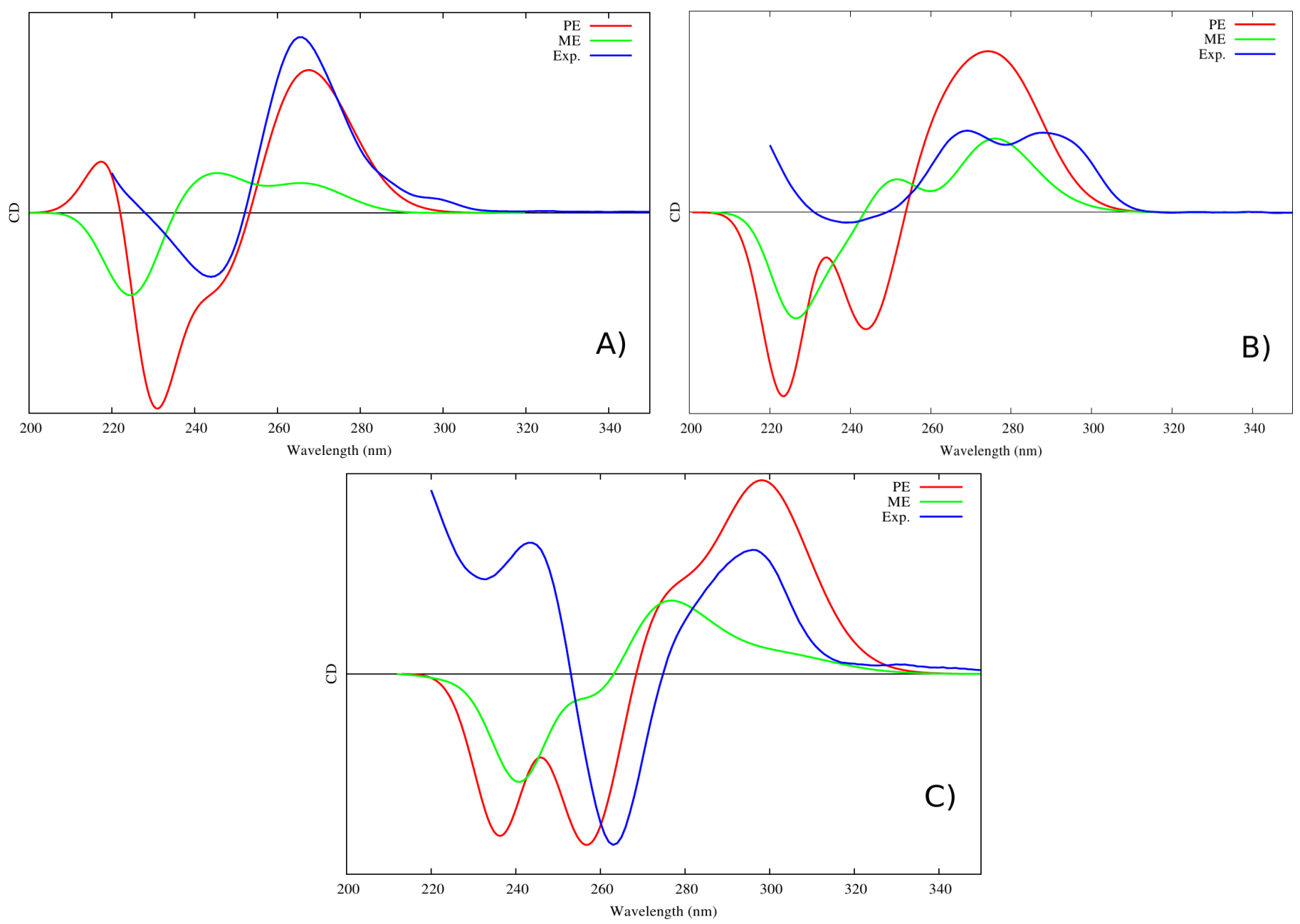

Figure 5: Comparison of $\mathrm{CD}$ spectra taking into account the environment using a polarizable embedding (red) ${ }^{2}$ and considering the mechanical embedding only (green), compared with the experimental spectrum (blue). The shift applied on each computed spectrum is the same for both computed spectra. A) Parallel, B) Hybrid and C) Antiparallel. 
V) Structural Parameters
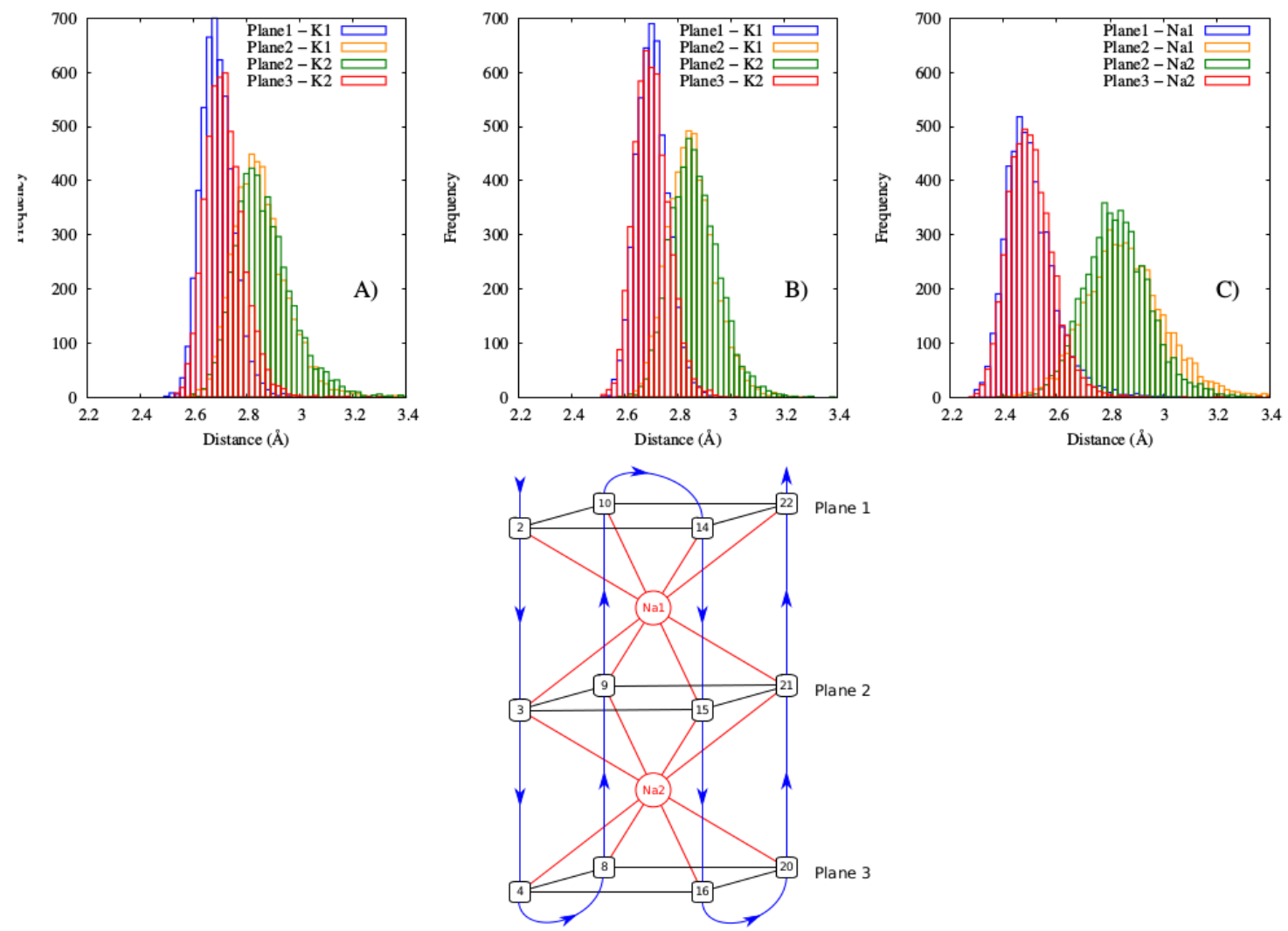

Figure 6: Distribution of the distances between the guanine center of mass and the stabilizing ions calculated along the MD trajectory. A) Parallel, B) Hybrid, C) Antiparallel. In the inlay we report the G4 schematic structure with the definition of the distances and of the planes. 
References

(1) Spata, V. A.; Matsika, S. Role of Excitonic Coupling and Charge-Transfer States in the Absorption and CD Spectra of Adenine-Based Oligonucleotides Investigated through QM/MM Simulations. J.

Phys. Chem. A 2014, 118, 12021-12030.

(2) Monari, A.; Assfeld, X.; Rivail, J. L. Theoretical Modeling of Large Molecular Systems. Advances in the Local Self Consistent Field Method for Mixed Quantum Mechanics/Molecular Mechanics Calculations Acc. Chem. Res., 2013, 46 (2), 596-603. 